\title{
NOTES ON EXACT MEETS AND JOINS
}

\author{
RICHARD N. BALL, JORGE PICADO, AND ALEŠ PULTR
}

Dedicated to George Janelidze on the occasion of his 60th birthday

\begin{abstract}
An exact meet in a lattice is a special type of infimum characterized by, inter alia, distributing over finite joins. In frames, the requirement that a meet is preserved by all frame homomorphisms makes for a slightly stronger property. In this paper these concepts are studied systematically, starting with general lattices and proceeding through general frames to spatial ones, and finally to an important phenomenon in Scott topologies.
\end{abstract}

\section{INTRODUCTION}

The notion of an exact meet probably first appeared in [7] (1970), under the name admissible meet. It was used as a technical device for the study of injective hulls of semilattices. In [2] (1984), the first author arrived at the concept by extrapolating the following characterization of meets in Boolean algebras. A lower bound $b$ of a subset $A$ in a Boolean algebra $B$ is the infimum of $A$ iff, for all differences $d-c$ in $B$,

$$
\forall a \in A(a \geqslant d-c \Rightarrow b \geqslant d-c .
$$

Modeling $x \geqslant d-c$ in a general lattice by $x \vee c \geqslant d$, and restricting this to the pairs $c<d$, one obtains the definition of a special type of meet called an exact meet, coinciding with the notion of an admissible meet, as it turned out. Such meets then proved useful for various purposes: injective hulls (again) and essential extensions, and in the study of completions of lattice ordered groups.

A very similar notion, with a very different motivation, appeared under the name free meet in [18] (1994). Free meets are the meets in frames which are

2010 Mathematics Subject Classification. 06D22, 18B35, 54D10.

Key words and phrases. Frame, locale, sublocale, lattice, exact meet, exact join, free meet, $T_{D}$-topological space, Scott topology, $P$-frame, paracompact frame.

Ball and Pultr were partially supported by a University of Denver PROF grant and by CEITI under the project P202/12/G061 of GAČR. Picado was partially supported by CMUC/FCT, through the program COMPETE/FEDER, and grant MTM2012-37894-C02-02 of the Ministry of Economy and Competitiveness of Spain. 
preserved by all frame homomorphisms. The property of being free is stronger than exactness (and we use the term strong exactness here) but it is closely related.

To illustrate the situation, consider the lattice $\Omega(X)$ of all open subsets of a $T_{D^{-}}$ topological space $X$. Here, the exact meets are those intersections which happen to be open, in other words the meets that coincide with intersections. Because

such meets are intersections, they distribute over all finite joins with elements of $\Omega(X)$ and in fact, this is the characteristic feature of exact meets in general. In a general space $X$, strongly exact meets of open sets are always open intersections, but if $X$ is not $T_{D}$ then there are intersections that are not open such that their meet is exact nevertheless.

Open intersections of systems of open sets also appeared in another context, in an important step in proving the Hofmann-Lawson duality. Namely, in the Scott topology of a continuous frame, the intersection of certain open sets was shown to be open iff the open sets were indexed by a compact index set. (For a precise formulation see 5.2 below.)

In this paper we present a systematic study of these phenomena. We start with exactness in a general, not necessarily distributive, lattice. Then we proceed to frames, where we are dealing with the exactness of the meets only, since all joins are exact in frames. (The latter feature, by the way, distinguishes frames among complete lattices.) We obtain characterizations in terms of the behavior of closed and open sublocales; in fact, the characterizations for general lattices in the preceding section can be viewed as describing the behavior of 'generalized closed sublocales'. The discrepancy between the characterizations in terms of the open sublocales as opposed to the closed ones then leads to the reappearance of the aforementioned free meets of T. Wilson. Furthermore, the situation is analyzed in the case of spatial frames, where the $T_{D}$-spatiality makes the two notions coincide, and is, in fact, characterized by this fact. Finally, we discuss the open intersections in Scott topologies. We conclude with a brief discussion of the preservation of exact meets by homomorphisms.

\section{Preliminaries and Problem Setting}

1.1. Although some of the statements may be formulated for more general posets, the most general setting we will consider will be lattices $L$ without special completeness or distributivity properties. For a subset $A$ and element $x$ of a lattice $L$, we shall write, as usual,

$$
\uparrow A=\{y \mid y \geqslant a \in A\}, \quad \text { and } \quad \uparrow x=\{y \mid y \geqslant x\} \text {. }
$$


We express the fact that $x$ is an upper bound of $A$ by writing

$$
A \leqslant x \quad \text { if } \quad \forall a \in A, a \leqslant x .
$$

Similarly we write $x \leqslant A$ if $x$ is a lower bound of $A$, and we make use of the abbreviations

$$
A \vee b=\{a \vee b \mid a \in A\}, \quad A \wedge b=\{a \wedge b \mid a \in A\}
$$

and

$$
A \rightarrow x=\{a \rightarrow x \mid a \in A\}
$$

From [2] we adopt the operations

$$
a \downarrow b=\{x \mid x \wedge b \leqslant a\} \quad \text { and } \quad a \uparrow b=\{x \mid x \vee a \geqslant b\}
$$

We write

$$
\bigvee A, \quad \operatorname{resp} . \bigwedge A
$$

for the supremum (join), resp. infimum (meet) of $A$ if it exists, so that use of the symbol entails the assertion that the supremum or infimum exists.

1.2. Recall that a frame is a complete lattice $L$ satisfying the distributivity law

$$
(\bigvee A) \wedge b=\bigvee(A \wedge b)=\bigvee_{a \in A}(a \wedge b)
$$

for all $A \subseteq L$ and $b \in L$. We speak of a co-frame if we have the distributivity law $(\bigwedge A) \vee b=\bigwedge(A \vee b)$ instead. Frame homomorphisms are maps preserving all joins and all finite meets; the resulting category will be denoted by Frm. A typical frame is the lattice $\Omega(X)$ of all open sets of a topological space; if $f: X \rightarrow Y$ is a continuous map then $\Omega(f)=\left(U \mapsto f^{-1}[U]\right): \Omega(Y) \rightarrow \Omega(X)$ is obviously a frame homomorphism.

Every frame is a Heyting algebra with Heyting operation $\cdot \rightarrow \cdot$ given by

$$
a \rightarrow b=\bigvee\{x \mid x \wedge a \leqslant b\} .
$$

In particular, a frame has pseudocomplements $a^{*}=a \rightarrow 0=\bigvee\{x \mid x \wedge a=0\}$. Similarly, a co-frame has pseudosupplements, i.e., co-pseudocomplements $a^{\#}=$ $\bigwedge\{x \mid x \vee a=1\}$. For more about frames see, e.g., $[13,17]$, or the more recent $[15]$. 
1.3. Sublocales. Frames can be viewed as generalized spaces. Subspaces of a frame $L$ are then represented as sublocales, that is, as subsets $S \subseteq L$ such that

- for all $M \subseteq S$, the meet $\bigwedge M$ lies in $S$, and

- for every $x \in L$ and $s \in S, x \rightarrow s$ lies in $S$.

The sublocale $S$ is a frame in the order inherited from $L$, and there is a natural frame surjection $L \rightarrow S$ (the representation of a "subspace" is contravariant), namely the left Galois adjoint $j^{*}$ of the embedding $j: S \rightarrow L$, which is a localic map in the sense of 1.3 .2 below. The family of all sublocales of $L$ constitutes a co-frame

$$
\mathcal{S} \ell(L)
$$

with intersection for meet, and join defined by

$$
\bigvee_{J} S_{i}=\left\{\bigwedge M \mid M \subseteq \bigcup_{J} S_{i}\right\}
$$

1.3.1. Open and closed sublocales. The open subspace of $L$ associated with the element $a \in L$ is represented by the open sublocale

$$
\mathfrak{o}(a)=\{a \rightarrow x \mid x \in L\}=\{x \mid a \rightarrow x=x\},
$$

which can be represented by the congruence $\Delta_{a}=\{(x, y) \mid x \wedge a=y \wedge a\}$.

The complement in $\mathcal{S} \ell(L)$ of $\mathfrak{o}(a)$ is the closed sublocale

$$
\mathfrak{c}(a)=\uparrow a,
$$

with the associated congruence $\nabla_{a}=\{(x, y) \mid x \vee a=y \vee a\}$. Note that the closure of a sublocale $S$, the smallest closed sublocale containing $S$, is given by a particularly simple formula

$$
\bar{S}=\operatorname{cl}(S)=\mathfrak{c}(\bigwedge S) .
$$

We recall from [15] the following equations in the co-frame $\mathcal{S} \ell(L)$ :

$$
\begin{array}{ll}
\bigvee_{J} \mathfrak{o}\left(a_{i}\right)=\mathfrak{o}\left(\bigvee_{J} a_{i}\right), & \mathfrak{o}(a) \wedge \mathfrak{o}(b)=\mathfrak{o}(a \wedge b), \\
\bigwedge_{J} \mathfrak{c}\left(a_{i}\right)=\mathfrak{c}\left(\bigvee_{J} a_{i}\right), & \mathfrak{c}(a) \vee \mathfrak{c}(b)=\mathfrak{c}(a \wedge b)
\end{array}
$$

1.3.2. Localic maps. Following the development of the second and third authors in their treatise [15], we will use the term localic map to refer to the right Galois adjoint $f: L \rightarrow M$ of a frame homomorphism $h: M \rightarrow L$; that is, we have $h(x) \leqslant y$ iff $x \leqslant f(y) .{ }^{1}$ That is, they are the meet-preserving maps $f: L \rightarrow M$ whose left

\footnotetext{
$1^{1}$ The reader is cautioned that the term "localic morphism" has a different meaning in topos theory. Here we have a concrete representation of a "morphism of locales" (a formal morphism in the category $\mathbf{L o c}=\mathbf{F r m}^{\mathrm{op}}$ ) as a map.
} 
adjoints $f^{*}: M \rightarrow L$ preserve binary meets. Alternatively, they may be described as the meet-preserving maps $L \rightarrow M$ which satisfy

$$
f\left(f^{*}(a) \rightarrow b\right)=a \rightarrow f(b) .
$$

See [15] for proofs and additional details. From this same text we shall also require several technical results in the sequel.

1.3.3. Lemma. Let $f: L \rightarrow M$ be a localic map with left adjoint $f^{*}: M \rightarrow L$.

(1) The image function $f[-]=(S \mapsto f[S])$ maps $\mathcal{S} \ell(L)$ into $\mathcal{S} \ell(M)$ and preserves joins.

(2) For each sublocale $T$ of $M$ there is a unique largest sublocale contained in $f^{-1}[T]$, designated $f_{-1}[T]$.

(3) The function $f_{-1}: \mathcal{S} \ell(M) \rightarrow \mathcal{S}(L)$ is right adjoint to the image function in (1), and therefore preserves all meets in $\mathcal{S}(M)$.

(4) $f_{-1}[\mathfrak{o}(a)]=\mathfrak{o}\left(f^{*}(a)\right)$ for all $a \in M$.

Proof. See II.2.3, III.4.1, and III.6.3 in [15].

1.3.4. The Booleanization sublocale. By the formula for closure, a sublocale $S \subseteq L$ is dense, that is, $\bar{S}=L$, iff $0 \in S$. Thus, every frame $L$ contains the minimal dense sublocale

$$
\mathfrak{B} L=\left\{a^{* *} \mid a \in L\right\}
$$

([12]). $\mathfrak{B} L$ is a Boolean frame, called the Booleanization of $L$.

1.4. Exact meets and joins. Recall [2]. An element $b$ is the exact meet of a subset $A$ of a lattice $L$ if

- $b$ is a lower bound of $A$, and

- for any $c<d$, if $A \subseteq c \uparrow d$ then $b \in c \uparrow d$.

(The latter in detail: if $a \vee c \geqslant d$ for all $a \in A$ then $b \vee c \geqslant d$.)

Dually, $b$ is the exact join of a subset $A$ if

- $b$ is an upper bound of $A$, and

- for any $c<d$, if $A \subseteq c \downarrow d$ then $b \in c \downarrow d$.

Exact meets and joins have various motivations. For instance, the exact joins and meets in a distributive lattice $L$ are precisely those which remain valid in the injective Boolean hull $\rho L$ of $L$ ([2, Proposition 1.10]). Here we will be particularly interested in the distributivity aspects of exact joins. In [7], subsets $A$ with exact joins are called admissible.

1.4.1. Proposition. An exact meet is a meet. 
Proof. Suppose $b$ is an exact meet of $A$ and consider an arbitrary $x \leqslant A$. Then $A \subseteq b \uparrow(b \vee x)$ implies $b \in b \uparrow(b \vee x)$, which is to say that $b \geqslant x$.

1.4.2. Theorem (Lemma $1.8[2])$. The following statements about an element $b$ and a subset $A$ of a lattice $L$ are equivalent.

(1) $b$ is an exact meet of $A$.

(2) $\bigwedge(A \vee x)=b \vee x$ for each $x$.

Proof. (1) $\Rightarrow(2): b \vee x$ is certainly a lower bound of $A \vee x$, and if it is not the greatest such then there is some $y \leqslant A \vee x$ such that $y \$ b \vee x$. Then we would have $b \vee x<y \vee b \vee x \leqslant A \vee x$, i.e., $A \subseteq(b \vee x) \uparrow(y \vee b \vee x)$, which would imply the contradiction $b \vee b \vee x=b \vee x \geqslant y \vee b \vee x$.

$(2) \Rightarrow(1)$ : Suppose $\bigwedge(A \vee x)=b \vee x$ for all $x$. For each $a \in A$ we get $b \leqslant a$ by setting $x=a$. If for $c<d$ we have $a \vee c \geqslant d$ for all $a \in A$ then $b \vee c=\bigwedge(A \vee c) \geqslant d$. This shows that $b$ is the exact meet of $A$.

It is a surprising fact that the property that every existing meet in a lattice is exact is expressible in the first order language of lattice theory.

1.4.3. Proposition (Proposition $1.10[2]$ ). The following statements about a lattice $L$ are equivalent.

(1) Every meet which exists in $L$ is exact.

(2) If $\bigwedge A$ exists for some subset $A \subseteq L$ then $\bigwedge(A \vee b)$ exists for every $b \in L$, and $\bigwedge(A \vee b)=(\bigwedge A) \vee b$.

(3) For all $a<b<c$ in $L$ there exists some $d \in L$ such that $a<d \leqslant c$ and $b \uparrow c \subseteq a \uparrow d$.

Consequently, such a lattice is distributive, and a co-frame if complete.

Proof. The equivalence of (1) and (2) is an immediate application of 1.4.2. So suppose these conditions hold in $L$, and consider $a<b<c$ for which no element $d$ can be found to satisfy (3). We claim that $\bigwedge A=a$, where $A=a \vee(b \uparrow c)$. For the claim could fail only if $a<d \leqslant A$ for some $d \in L$, in which case $b \uparrow c \subseteq a \uparrow d$, contrary to assumption. But $a$ is not the exact meet of $A$, for $A \subseteq b \uparrow c$ while $a \notin b \uparrow c$. This cannot happen by (2), so we are forced to conclude that an element $d$ can be found satisfying (3).

Suppose (1) fails, say $\bigwedge A=a$ but $A \vee b \geqslant c>a \vee b$. By replacing $b$ with $a \vee b$ if necessary, we may assume that $a<b<c$. Since $A \subseteq b \uparrow c$ and $\bigwedge A=a$, no element $d$ satisfying (3) can be found.

1.4.4. Remark. The concept of an exact join is, in a way, dual to, or, rather, orthogonal to, that of being a linear element of a lattice $L$. In [12], $a$ is called 
linear in $L$ if, for each $B \subseteq L$,

$$
a \wedge \bigvee B=\bigvee(a \wedge B)
$$

Dually, $a$ is termed co-linear if $a \vee \wedge B=\bigwedge(a \vee B)$ for all $B \subseteq L$. One of the notable facts is that, in a subfit frame, $a$ is co-linear if and only if it is complemented (see, e.g. $[12,15])$.

\section{ExACT MEETS IN GENERAL LATTICES}

In the sequel we will concentrate on exact meets. The results are easily dualized, and, more specifically, we will be interested in the phenomena in frames and particularly in topological spaces, where the joins are automatically exact and hence the exactness of meets is what is of interest.

2.1. In spaces and, as we shall see, in frames, the exactness of a meet is connected with the openness of an intersection of open objects and the closedness of the union of closed objects. For instance, if $U_{i}$ are open in $X$ and it so happens that $\bigcap U_{i}$ is open, then, for any open $V,\left(\bigcap U_{i}\right) \cup V=\bigcap\left(U_{i} \cup V\right)$ and hence $\wedge U_{i}=\bigcap U_{i}$ is an exact meet in $\Omega(X)$.

The general phenomena go in this direction. Therefore we will imitate the sublocale terminology and speak of the subsets of a general lattice of the form

$$
\mathfrak{c}(a)=\uparrow a
$$

as being the closed ones.

More generally, we define a geometric subset of a lattice $L$ (the lattices will be assumed bounded, but this may not be necessary) as a subset $S \subseteq L$ such that

$$
\text { if } M \subseteq S \text { and } \bigwedge M \text { exists then } \bigwedge M \in S
$$

The set of all geometric subsets of a lattice $L$ will be denoted by

$$
\mathcal{G}(L)
$$

2.2. Proposition. For any lattice $L, \mathcal{G}(L)$ is a complete lattice in the inclusion order, with the join given by

$$
\bigvee_{J} S_{i}=\left\{\bigwedge M \mid M \subseteq \bigcup_{J} S_{i}, \bigwedge M \text { exists }\right\}
$$

Consequently, if $L$ is a frame then the sublocale frame $\mathcal{S}(L)$ is a subset of $\mathcal{G}(L)$ closed under all joins. 
Proof. Let $N$ be a subset of $\bigvee S_{i}$ and let $\bigwedge N$ exist. For each $n \in N$ we have an $M_{n} \subseteq \bigcup S_{i}$ such that $\bigwedge M_{n}$ exists and is equal to $n$. Now it is a standard fact that then $\bigwedge\left(\bigcup\left\{M_{n} \mid n \in N\right\}\right)$ exists and is equal to $\bigwedge N$. If, in addition, $L$ is a frame and the $S_{i}$ 's are sublocales then for $x \in \bigvee S_{i}$, say $x=\bigwedge N$ for $N \subseteq \bigcup S_{i}$, and for $y \in L$ we have

$$
y \rightarrow x=y \rightarrow \bigwedge N=\bigwedge_{N}(y \rightarrow n)
$$

Since, for each $n \in N, y \rightarrow n \in \bigcup_{J} S_{i}$ because $n \in \bigcup_{J} S_{i}$, it follows that $y \rightarrow x \in$ $\bigvee_{J} S_{i}$ and consequently that $\bigvee_{J} S_{i} \in \mathcal{S} \ell(L)$.

2.3. Proposition. Let $\bigvee_{A} \mathfrak{c}(a)$ be closed in $\mathcal{G}(L)$. Then $\bigwedge A$ exists, and $\bigvee_{A} \mathfrak{c}(a)=$ $\mathfrak{c}(\bigwedge A)$.

Proof. Suppose $\bigvee_{A} \mathfrak{c}(a)=\uparrow b$. Then, first, all the $a$ 's are in $\uparrow b$ and hence $b \leqslant A$. If $x \leqslant A$ then $x$ is a lower bound of each subset $M \subseteq \uparrow A$. In particular, $b \in \bigvee_{A} \mathfrak{c}(a)$ entails $b=\bigwedge M$ for some $M \subseteq \uparrow A$, hence $x \leqslant b$.

2.4. Theorem. A meet $\bigwedge A$ is exact in $L$ if and only if the join $\bigvee_{A} \mathfrak{c}(a)$ is closed in $\mathcal{G}(L)$.

Proof. Let $u$ be the exact meet of $A$ in $L$. Surely $\uparrow u \supseteq \uparrow a$ for all $a \in A$, so that $\uparrow u \geqslant \bigvee_{A} \mathfrak{c}(a)$. To demonstrate the opposite inequality, consider an arbitrary $x \geqslant u$. Then we have by the exactness

$$
x=u \vee x=(\bigwedge A) \vee x=\bigwedge(A \vee x) .
$$

Since $A \vee x \subseteq \uparrow A=\bigcup_{A} \uparrow a$, this yields $x=\bigwedge(A \vee x) \in \bigvee_{A} \mathfrak{c}(a)$.

Let $\bigvee_{A} \uparrow a$ be closed, i.e., equal to $\uparrow u$ for some $u \in L$. Then $u=\bigwedge A$ by 2.3. To show this meet exact, consider an arbitrary $x \in L$. Then

$$
(\bigwedge A) \vee x=u \vee x \in \uparrow u
$$

and hence $u \vee x=\bigwedge B$ for some $B \subseteq \uparrow A$. That means that for each $b \in B$ there is some $a_{b} \in A$ such that $b \geqslant a_{b}$, and since $u \vee x \leqslant b$ we have $b \geqslant x$ and finally $b \geqslant a_{b} \vee x$. Thus,

$$
u \vee x=\bigwedge B \geqslant \bigwedge_{B}\left(a_{b} \vee x\right) \geqslant \bigwedge(A \vee x) \geqslant u \vee x .
$$

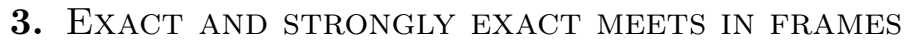

From now on, $L$ will be a frame. 
3.1. Recall that for the pseudosupplement $x^{\#}$ in a co-frame we have

$$
\begin{aligned}
y \geqslant\left(\bigwedge_{J} x_{i}\right)^{\#} & \Longleftrightarrow y \vee\left(\bigwedge_{J} x_{i}\right)=\bigwedge_{J}\left(y \vee x_{i}\right)=1 \Longleftrightarrow \forall i\left(y \vee x_{i}=1\right) \\
& \Longleftrightarrow \forall i\left(y \geqslant x_{i}^{\#}\right)
\end{aligned}
$$

and hence

$$
\left(\bigwedge_{J} x_{i}\right)^{\#}=\bigvee_{J} x_{i}^{\#}
$$

3.2. Lemma. In the co-frame $\mathcal{S} \ell(L)$ we have $\bigvee_{J} \mathfrak{c}\left(a_{i}\right)=\mathfrak{c}(a)$ if and only if $\left(\bigcap_{J} \mathfrak{o}\left(a_{i}\right)\right)^{\# \#}=\mathfrak{o}(a)$.

Proof. Set $S=\bigcap_{J} \mathfrak{o}\left(a_{i}\right)$ and let $S^{\# \#}=\mathfrak{o}(a)$. Then by (3.1.1)

$$
\bigvee \mathfrak{c}\left(a_{i}\right)=\bigvee \mathfrak{o}\left(a_{i}\right)^{\#}=\left(\bigwedge \mathfrak{o}\left(a_{i}\right)\right)^{\#}=S^{\#}=S^{\# \# \#}=\mathfrak{o}(a)^{\#}=\mathfrak{c}(a) .
$$

On the other hand, if $\bigvee \mathfrak{c}\left(a_{i}\right)=\mathfrak{c}(a)$ then, again by (3.1.1),

$$
\left(\bigwedge \mathfrak{o}\left(a_{i}\right)\right)^{\# \#}=\left(\left(\bigwedge \mathfrak{o}\left(a_{i}\right)\right)^{\#}\right)^{\#}=\left(\bigvee \mathfrak{o}\left(a_{i}\right)^{\#}\right)^{\#}=\left(\bigvee \mathfrak{c}\left(a_{i}\right)\right)^{\#}=\mathfrak{c}(a)^{\#}=\mathfrak{o}(a)
$$

From $1.4 .2,2.2,2.4$, and 3.2 we immediately obtain

3.3. Theorem. The following facts about a meet $a=\bigwedge_{J} a_{i}$ in a frame $L$ are equivalent.

(1) The meet $a=\bigwedge_{i} a_{i}$ is exact.

(2) For every $b \in L, \bigwedge_{i}\left(a_{i} \vee b\right)=a \vee b$.

(3) $\bigvee_{i} \mathfrak{c}\left(a_{i}\right)=\mathfrak{c}(a)$ in $\mathcal{S} \ell(L)$.

(4) $\bigvee_{i} \mathfrak{c}\left(a_{i}\right)$ is a closed sublocale of $L$.

(5) If $x \geqslant a$ then there exist $x_{i} \geqslant a_{i}$ such that $x=\bigwedge_{i} x_{i}$.

(6) $\left(\bigwedge_{i} \mathfrak{o}\left(a_{i}\right)\right)^{\# \#}=\left(\bigcap_{i} \mathfrak{o}\left(a_{i}\right)\right)^{\# \#}=\mathfrak{o}(a)$ in $\mathcal{S} \ell(L)$.

(7) $\left(\bigwedge_{i} \mathfrak{o}\left(a_{i}\right)\right)^{\# \#}=\left(\bigcap_{i} \mathfrak{o}\left(a_{i}\right)\right)^{\# \#}$ is an open sublocale of $L$.

((5) is just $\bigvee_{j} \uparrow a_{i}=\uparrow a$ explicitly rewritten.)

3.4. Characterizing $P$-frames. Of some importance in general topology are the $P$-spaces, i.e., the Tychonoff spaces on which a continuous real-valued function must be constant in some neighborhood of each point. Zero sets are obviously open in such spaces, meaning cozero sets are clopen. Indeed, this is taken as the frame definition: a completely regular frame $L$ is said to be a $P$-frame if each cozero element is complemented, i.e., if its cozero part $\operatorname{coz} L$ is a Boolean $\sigma$-frame. (See 
[3] for several characterizations of $P$-frames, together with information on their role in the general theory.)

Perhaps the handiest of the several well-known characterizations of $P$-spaces is that a countable intersection of open sets remains open. But this attribute has resisted a pointfree formulation, and for good reason. To say of an open set that it is the set-theoretic intersection of some countable family in the frame of open sets is much stronger than to say that it is their meet. In fact, this strict sort of meet would appear at first glance to be an inherently pointed notion.

Notice, however, the meets which are actually set-theoretic intersections are just those that commute with the joins. On this basis, one might therefore hope to capture the $P$-frame property by requiring countable meets to be exact. Such is not the case, unfortunately, but a slightly weaker condition does work. We shall say that a meet $a=\bigwedge a_{i}$ is cozero exact if $\bigwedge\left(a_{i} \vee b\right)=a \vee b$ for all $b \in \operatorname{coz} L$.

3.4.1. Theorem. A completely regular frame is a $P$-frame iff each countable meet is cozero exact.

Proof. Suppose each countable meet in $L$ is cozero exact. Consider a cozero element $a \in L$, and write $a=\bigvee_{n} a_{n}$ for cozero elements $a_{n} \prec a$. Then $a^{*}=\bigwedge_{n} a_{n}{ }^{*}$ and

$$
a \vee a^{*}=a \vee \bigwedge_{n} a_{n}^{*}=\bigwedge_{n}\left(a \vee a_{n}^{*}\right)=1
$$

which shows that $a$ is complemented.

Now suppose that $L$ is a $P$-frame, i.e., $a \vee a^{*}=1$ for all $a \in \operatorname{coz} L$. Consider an arbitrary subset $\left\{a_{n}\right\}$ and a cozero element $b$ of $L$. Since clearly $b \vee \bigwedge_{n} a_{n} \leqslant$ $\bigwedge_{n}\left(b \vee a_{n}\right)$, we need only establish the opposite inequality. For that purpose, observe that $\left(\bigwedge_{n}\left(b \vee a_{n}\right)\right) \wedge b^{*} \leqslant \bigwedge_{n} a_{n}$, and since $b$ is complemented, then $\bigwedge_{n}\left(b \vee a_{n}\right) \leqslant b \vee \bigwedge_{n} a_{n}$.

3.5. Strongly exact (free) meets. Points (3) and (4) in 3.3, compared with (6) and (7), give rise naturally to the question of what happens if we require open meets (intersections) of the open sublocales instead of closed joins of the corresponding closed sublocales, i.e.,

$$
\bigwedge_{J} \mathfrak{o}\left(a_{i}\right)=\bigcap_{J} \mathfrak{o}\left(a_{i}\right)=\mathfrak{o}(a)
$$

This property is stronger (see 3.5.4, 3.6.2 and 4.3.1 below). For our purposes, we will refer to the s-exact property as strong exactness, and immediately obtain

3.5.1. Theorem. The following facts about a meet $a=\bigwedge_{J} a_{i}$ in a frame $L$ are equivalent. 
(1) The meet $a=\bigwedge_{i} a_{i}$ is strongly exact.

(2) $\bigwedge_{i} \mathfrak{o}\left(a_{i}\right)=\bigcap_{i} \mathfrak{o}\left(a_{i}\right)$ is an open sublocale of $L$.

(3) If $a_{i} \rightarrow x=x$ for all $i \in J$ then $\left(\bigwedge_{J} a_{i}\right) \rightarrow x=x$.

(3) is just the s-exact condition written explicitly. Furthermore, if $\bigcap_{i} \mathfrak{o}\left(a_{i}\right)=$ $\mathfrak{o}(a)$ then, necessarily, $a=\bigwedge a_{i}$. This follows immediately from the fact that $\mathfrak{o}(x) \subseteq \mathfrak{o}(y)$ iff $x \leqslant y$.

3.5.2. Viewed from another perspective, strongly exact meets appeared under the name "free meets" in the unpublished thesis of Todd Wilson [18]. There they were defined as the meets which are preserved by all frame homomorphisms. Wilson characterized freeness by means of several interesting conditions, one of which was s-exactness. Here is a variant of Wilson's characterization.

3.5.3. Theorem. The following statements about a meet $\bigwedge_{J} a_{i}$ in a frame $L$ are equivalent.

(1) $\bigwedge_{i} a_{i}$ is strongly exact.

(2) For every frame homomorphism $h: L \rightarrow M, h\left(\bigwedge_{i} a_{i}\right)=\bigwedge_{i} h\left(a_{i}\right)$, and $\bigwedge_{i} h\left(a_{i}\right)$ is strongly exact.

(3) For every frame homomorphism $h: L \rightarrow M, h\left(\bigwedge_{i} a_{i}\right)=\bigwedge_{i} h\left(a_{i}\right)$.

(4) For every $x \in L, \bigwedge((A \rightarrow x) \rightarrow x)=((\bigwedge A) \rightarrow x) \rightarrow x$.

Proof. $(1) \Rightarrow(2)$ : Let $f$ be the localic map adjoint to a frame homomorphism $h: L \rightarrow M$. Consider $f_{-1}: \mathcal{S} \ell(L) \rightarrow \mathcal{S} \ell(M)$. By 1.3 .3 we have

$$
\bigcap \mathfrak{o}\left(h\left(a_{i}\right)\right)=\bigcap f_{-1}\left(\mathfrak{o}\left(a_{i}\right)\right)=f_{-1}(\mathfrak{o}(a))=\mathfrak{o}(h(a)) .
$$

$(2) \Rightarrow(3)$ is trivial.

$(3) \Rightarrow(4)$ : Consider the mapping $h_{x}: L \rightarrow \mathfrak{B c}(x)$ defined by

$$
h_{x}(a)=(a \rightarrow x) \rightarrow x .
$$

Since $a \rightarrow x$ is the pseudocomplement of $c(x)(a)=x \vee a$ in $\mathfrak{c}(x)=\uparrow x$, it is clear that $h_{x}$ is the frame map $b_{\mathfrak{c}(x)} \circ c(x)$. Thus $h_{x}$ preserves the meet of $A$ by assumption, and, since the meets in the frame $\mathfrak{B c}(x)$ coincide with the meets in $L$, the conclusion follows.

$(4) \Rightarrow(1)$ : By 3.5.1(3) it suffices to check that $a \rightarrow x=x$ for every $a \in A$ implies $(\bigwedge A) \rightarrow x=x$, i.e., $(\bigwedge A) \rightarrow x \leqslant x$, since the other inequality is always true. So let $a \rightarrow x=x$ for every $a \in A$. Then, by hypothesis, $((\bigwedge A) \rightarrow x) \rightarrow x=x \rightarrow x=1$ and hence $(\bigwedge A) \rightarrow x \leqslant x$.

3.5.4. Example. Consider the topological space

$$
X=(\{1,2, \ldots, n, \ldots\},\{\varnothing\} \cup\{\tilde{n} \mid n=1,2, \ldots\})
$$


where $\tilde{n}=\{k \mid k \geqslant n\}$. Then its topology $L$ is obviously both a frame and a co-frame so that all meets in $L$ are exact. On the other hand we have the frame homomorphism $h: L \rightarrow\{0,1\}$ with $h(\varnothing)=0$ and $h(\tilde{n})=1$ otherwise. Now $h\left(\bigwedge_{n} \tilde{n}\right)=h(\varnothing)=0 \neq 1=\bigwedge_{n} h(\tilde{n})$ and hence $\bigwedge_{n} \tilde{n}$ is not strongly exact.

3.6. Conservative subsets. In [9], the authors use conservative subsets of frames to study paracompactness. Translated into our language, a subset $A \subseteq L$ is conservative if $\bigwedge B$ is exact for every $B \subseteq A$. Chen [8] also uses conservative sets to present some new characterizations of paracompact frames. In particular, he proves characterization (4) in our 3.3 using congruences ([8, Lemma 2.3]).

Exact meets are also related to the concepts of interior-preserving and closurepreserving families of sublocales of Plewe ([16]). Recall that a family $\mathcal{S}=\left\{S_{i} \mid i \in\right.$ $I\} \subseteq \mathcal{S} \ell(L)$ is closure-preserving if for all $J \subseteq I, \operatorname{cl}\left(\bigvee_{J} S_{i}\right)=\bigvee_{J} \mathrm{cl}\left(S_{i}\right)$. Dually, $\mathcal{S}$ is interior-preserving if for all $J \subseteq I$, int $\left(\bigwedge_{J} S_{i}\right)=\bigwedge_{J}$ int $\left(S_{i}\right)$. Then, a subset $A$ of $L$ is said to be interior-preserving (resp. closure-preserving) if $\{\mathfrak{o}(a) \mid a \in A\}$ is interior-preserving (resp. $\{\mathfrak{c}(a) \mid a \in A\}$ is closure-preserving). Interior-preserving covers play a decisive role in the construction of canonical examples of transitive quasi-uniformities for frames ([10]). Of course, any interior-preserving cover of $L$ is closure-preserving but, somewhat surprisingly and contrary to what happens in spaces, the converse does not hold in general.

3.6.1. Lemma. Let $A \subseteq L$. Then:

(1) $A$ is interior-preserving iff $\bigwedge_{B} \mathfrak{o}(b)=\mathfrak{o}(\bigwedge B)$ for every $B \subseteq A$.

(2) $A$ is closure-preserving iff $\bigvee_{B} \mathfrak{c}(b)=\mathfrak{c}(\bigwedge B)$ for every $B \subseteq A$.

Proof. We only prove (a), the proof for (b) is similar. $A$ is interior-preserving iff $\{\mathfrak{o}(a) \mid a \in A\}$ is interior-preserving iff int $\left(\bigwedge_{B} \mathfrak{o}(b)\right)=\bigwedge_{B}$ int $(\mathfrak{o}(b))$ for every $B \subseteq A$ iff int $\left(\bigwedge_{B} \mathfrak{o}(b)\right)=\bigwedge_{B}(\mathfrak{o}(b))$ for every $B \subseteq A$ iff $\bigwedge_{B} \mathfrak{o}(b)$ is open for every $B \subseteq A$.

From Lemma 3.6.1 and Theorem 3.3 we immediately obtain

3.6.2. Corollary. A subset $A$ of a frame $L$ is conservative if and only if it is closure-preserving.

\section{Exact meets in spaCes and spatial frames}

4.1. $T_{D}$ and $T_{D-0}$. Recall that a space $X$ is $T_{D}$ if

$$
\forall x \in X \exists U \ni x \text { open such that } U \backslash\{x\} \text { is open. }
$$

(This concept goes back to 1963, see [1] and [6].) More generally, a space $X$ is $T_{D-0}$ if its $T_{0}$-modification $X^{0}$ is $T_{D}$. The $T_{0}$-modification $X^{0}$ of a space $X$ is obtained 
by factoring $X$ by the equivalence

$$
x \sim y \quad \equiv_{\mathrm{df}} \quad \overline{\{x\}}=\overline{\{y\}} .
$$

We will need the notion of a $\sim$-set in $X$, namely a subset $A \subseteq X$ such that

$$
x \in A \text { and } x \sim y \Rightarrow y \in A .
$$

Obviously each open set is a $\sim$-set.

4.2. Proposition. A space is $T_{D}$ iff the following equivalence holds.

$$
(\forall A \text { open, int }(U \cup A)=\operatorname{int} U \cup A) \quad \text { iff } U \text { is open. }
$$

Proof. Let $X$ be $T_{D}$ and let $\operatorname{int}(U \cup A)=\operatorname{int} U \cup A$ for all open $A$. Let $x \in U$. Choose an open $A$ such that $x \notin A$ and $A \cup\{x\}$ is open. Then int $(U \cup A)=\operatorname{int} U \cup A$ and hence $x \in \operatorname{int} U$.

Let the implication hold and let $x \in X$. If $U \cup\{x\}$ is open for every open $U$ there is nothing to prove. Else choose an open $U$ such that $U \cup\{x\}$ is not open. By the implication there is an open $A$ such that int $(U \cup\{x\} \cup A) \neq \operatorname{int}(U \cup\{x\}) \cup A=$ $U \cup A$. Obviously $x$ is the only element in which the two sets can differ, and hence $x \in \operatorname{int}(U \cup\{x\} \cup A)$ and there is an open $V$ such that $x \in V \subseteq U \cup\{x\} \cup A$. Then $V \backslash\{x\}=V \cap(U \cup A)$ is open.

4.2.1. Corollary. A space is $T_{D-0}$ iff the following equivalence holds for every $\sim$ set $U$.

$$
(\forall A \text { open, } \operatorname{int}(U \cup A)=\operatorname{int} U \cup A) \quad \text { iff } \quad U \text { is open. }
$$

4.2.2. Lemma. In any space $X$,

$$
\operatorname{int} U=\bigwedge\{X \backslash \overline{\{x\}} \mid x \notin U\} \text {. }
$$

Proof. An open $V \subseteq U$ is a subset of each $X \backslash \overline{\{x\}}$ with $x \notin U$; hence $V \subseteq$ $\bigwedge\{X \backslash \overline{\{x\}} \mid x \notin U\}$. On the other hand, $\bigwedge_{x \notin U}(X \backslash \overline{\{x\}})$ is open and we have

$$
\bigwedge_{x \notin U}(X \backslash \overline{\{x\}}) \subseteq \bigcap_{x \notin U}(X \backslash \overline{\{x\}}) \subseteq \bigcap_{x \notin U}(X \backslash\{x\})=U .
$$

Thus, $\bigwedge\{X \backslash \overline{\{x\}} \mid x \notin U\}$ is the largest open set contained in $U$.

4.2.3. Theorem. The following statements are equivalent for a topological space $X$.

(1) $X$ is $T_{D-0}$.

(2) A meet $\wedge U_{i}$ is exact in $\Omega(X)$ iff $\bigcap U_{i}$ is open. 
Proof. (1) $\Rightarrow(2)$ : Obviously if $\bigcap U_{i}$ is open then $\bigwedge U_{i}$ is exact. Now let $\wedge U_{i}$ be exact. Set $U=\bigcap U_{i}$ and take any open $A$. We have

$$
\begin{aligned}
\operatorname{int} U \cup A & =\left(\bigwedge U_{i}\right) \cup A=\bigwedge\left(U_{i} \cup A\right)=\operatorname{int} \bigcap\left(U_{i} \cup A\right)= \\
& =\operatorname{int}\left(\left(\bigcap U_{i}\right) \cup A\right)=\operatorname{int}(U \cup A)
\end{aligned}
$$

and hence, since $U$ is obviously a $\sim$-set (all the $U_{i}$ are), $U$ is open by 4.2.1.

$(2) \Rightarrow(1)$ : We will prove that the equivalence from the display in 4.2.1 holds. Let $U$ be a $\sim$-set such that for any open $A$

$$
\operatorname{int}(U \cup A)=\operatorname{int} U \cup A .
$$

Recall 4.2.2. We have

$$
\begin{aligned}
\bigwedge\{X \backslash \overline{\{x\}} \mid x \notin U\} \cup A=\operatorname{int} U \cup A=\bigwedge\{X \backslash \overline{\{x\}} \mid x \notin U \cup A\}= \\
=\bigwedge\{(X \backslash \overline{\{x\}}) \cup A \mid x \notin U \cup A\} \supseteq \bigwedge\{(X \backslash \overline{\{x\}}) \cup A \mid x \notin U\} .
\end{aligned}
$$

(The last equality holds since $X \backslash \overline{\{x\}} \supseteq A$ if $x \notin A$.) This makes int $U=$ $\bigwedge\{X \backslash \overline{\{x\}} \mid x \notin U\}$ an exact meet and consequently makes $U$ open.

4.2.4. Corollary. In a $T_{D-0}$ space $X$, each meet is exact iff $X$ is quasidiscrete (i.e., the intersection of any family of open sets is open).

4.3. Proposition. Let $X$ be a general topological space and let $\bigwedge_{i} U_{i}$ be strongly exact in $\Omega(X)$. Then $\bigcap_{i} U_{i}$ is open.

Proof. Consider the congruences $\Delta_{U_{i}}, \Delta_{U}$ from 1.3.1. If $\mathfrak{o}(U)=\bigcap \mathfrak{o}\left(U_{i}\right)$ then $\Delta_{U}$ is the supremum of the system of congruences $\Delta_{U_{i}}$ in the lattice of congruences on $L$, which is dually isomorphic to $\mathcal{S}(L)$. Set $A=\bigcap_{i} U_{i}$ and consider the congruence

$$
E=\{(V, W) \mid V, W \in \Omega(X), V \cap A=W \cap A\} .
$$

If $V \cap U_{i}=W \cap U_{i}$ then $V \cap A=V \cap U_{i} \cap A=W \cap U_{i} \cap A=W \cap A$, hence $\Delta_{U_{i}} \subseteq E$ for all $i$, and hence $\Delta_{U} \subseteq E$. In particular $U \Delta_{U} X$ and hence $U E X$, that is, $U \cap A=A$ and $A \subseteq U$, and since $U$ is the interior of $A, A=U$.

4.3.1. Note. On the other hand, $\bigcap U_{i}$ can be open without $\wedge U_{i}$ being strongly exact. Recall Example 3.5.4. We have $\bigcap_{n \geqslant 1} \tilde{n}=\varnothing$, hence open, while $\bigwedge_{n \geqslant 1} \tilde{n}$ is not strongly exact.

Thus, the property of $\bigcap U_{i}$ being open in $\Omega(X)$ is in general strictly between exactness and strong exactness. Here is a large class of spaces in which the open intersections and strongly exact meets do not coincide. 
Observation. Let $X$ be a non-empty $T_{1}$-space without isolated points. Then $\bigcap_{x \in X}(X \backslash\{x\})=\varnothing$ is open but $\bigwedge_{x \in X}(X \backslash\{x\}) \supseteq \mathfrak{B} \Omega(X)$ (recall 1.3.4: all the $X \backslash x$ are dense) and hence it is not strongly exact.

\section{Open intersections in Scott topology}

Scott topologies are typically not $T_{D}$ and hence the first part of Section 4 does not apply. We will discuss the open intersections only.

5.1. The set of all up-sets (that is, the $M \subseteq X$ such that $\uparrow M=M$ ) of a poset $X$ will be denoted by

$$
\mathfrak{U}(X) .
$$

Recall that the Scott topology $\sigma_{X}$ on a poset $X$ with suprema of directed sets consists of the $U \in \mathfrak{U}(X)$ such that

$$
\text { for any directed } D \subseteq X, \quad \bigvee D \in U \Rightarrow D \cup U \neq \varnothing
$$

In this section the spectrum of a frame $L$ will be represented as the set $\Sigma^{\prime} L$ of all completely prime filters $P$ in $L$ endowed with the topology consisting of the open sets $\Sigma_{a}^{\prime}=\{P \mid a \in P\}, a \in L$. It is a well known (and very easy) fact that each $P \in \Sigma^{\prime} L$ is Scott open in $L$.

More generally, in a general lattice $L$ we will consider the pre-topology

$$
\Sigma_{L}^{\prime}=\left\{\Sigma_{x}^{\prime} \mid x \in L\right\}, \quad \Sigma_{x}^{\prime}=\{U \in \mathfrak{U}(L) \mid x \in U\} .
$$

5.2. One of the important facts needed in the proof of the Hofmann-Lawson duality $([11]$, see also $[13,17,15])$ is that

an intersection $\bigcap \mathcal{P}$ of a set of completely prime filters is Scott open iff $\mathcal{P}$ is a compact subset of $\Sigma^{\prime} L$.

In this section we will show that this is part of a more general fact.

5.3. A subset $\mathcal{U}$ of $\mathfrak{U}(L)$ will be called $d$-compact if one can choose in every directed cover of $\mathcal{U}$ by the element of $\Sigma_{L}^{\prime}$ an element covering $\mathcal{U}$.

5.3.1. Proposition. Let a set $\mathcal{U}$ of $S$ cott open sets be d-compact in $\Sigma_{L}^{\prime}$. Then $\cap \mathcal{U}$ is Scott open.

Proof. Take an $s=\bigvee D \in \bigcap \mathcal{U}$ with $D$ directed. Then $s \in U$ for each $U \in \mathcal{U}$ and hence there is a $d(U) \in D$ such that $d(U) \in U$, that is, $U \in \Sigma_{d(U)}^{\prime}$.

For $\left(U_{1}, \ldots, U_{n}\right)$ choose $d\left(U_{1}, \ldots, U_{n}\right) \in D, d\left(U_{1}, \ldots, U_{n}\right) \geqslant U_{i}, i=1, \ldots, n$. Then $U_{i} \in \Sigma_{d\left(U_{1}, \ldots, U_{n}\right)}^{\prime}$ for all $i$, and

$$
C=\left\{\Sigma_{d\left(U_{1}, \ldots, U_{n}\right)}^{\prime} \mid U_{1}, \ldots, U_{n} \in \mathcal{U}\right\}
$$


is a directed cover of $\mathcal{U}$. By d-compactness we have a $d \in C(\subseteq D)$ such that $U \subseteq \Sigma_{d}^{\prime}$ for all $U \in \mathcal{U}$ so that $d \in \bigcap \mathcal{U}$.

5.3.2. Proposition. Let $X=(X, \leqslant)$ be a complete lattice. Let $\mathcal{U}$ be a set of Scott open sets in $X$ and let $\bigcap \mathcal{U}$ be Scott open. Then $\mathcal{U}$ is d-compact in $\Sigma_{X}^{\prime}$.

Proof. Let $\mathcal{U} \subseteq \bigcup\left\{\Sigma_{d}^{\prime} \mid d \in D\right\}$ with $D$ directed such that $U_{d} \subseteq U_{e}$ whenever $d \leqslant e$. Then for each $U \in \mathcal{U}$ there is a $d \in D$ such that $U \in \Sigma_{d}^{\prime}$, hence $d \in U$ so that $\bigvee D \in U$ and finally $\bigvee D \in \bigcap \mathcal{U}$. Since $\bigcap \mathcal{U}$ is Scott open there is a $d \in D$ such that $d \in \bigcap \mathcal{U}$, and hence $U \subseteq \Sigma_{d}^{\prime}$ for all $U \in \mathcal{U}$.

5.4. Proposition. Let $L$ be a complete lattice. Then the intersection $\bigcap \mathcal{U}$ in the Scott topology $\sigma_{L}$ is open iff $\mathcal{U}$ is d-compact in the pretopology $\Sigma_{L}^{\prime}$ on $\mathfrak{U}(L)$.

\section{More ABOUt EXACTNESS AND MAPS}

In 3.4 we saw that each frame homomorphism preserves all strongly exact meets. Indeed this fact characterized strong exactness. Consequently, no such universal behaviour can be expected from plain exactness. In this section we will present two special facts.

First, however, we will apply 3.5 to the $T_{D}$ case. From 4.2 .4 we immediately obtain

6.1. Corollary. If $L$ is $T_{D}$-spatial then a frame homomorphism $h: L \rightarrow M$ sends all exact meets in $L$ to strongly exact meets in $M$.

6.2. Recall from [4] that a frame homomorphism $h$ is weakly open if

$$
h\left(x^{* *}\right) \leqslant h(x)^{* *} .
$$

We have

6.2.1. Proposition. Let $h$ be a frame homomorphism such that for the associated localic map $f$ the preimage homomorphism $f_{-1}$ between the frames of congruences is weakly open. Then $h$ preserves all exact meets.

Proof. In the language of the co-frames of sublocales (dual to the frame of congruences resp. nuclei) we assume that

$$
f_{-1}(S)^{\# \#} \subseteq f_{-1}\left(S^{\# \#}\right) .
$$

Let $a=\bigwedge_{i} a_{i}$ be an exact meet. By 3.3(6), o $(a)=\left(\bigwedge_{i} \mathfrak{o}\left(a_{i}\right)\right)^{\# \#}$. Writing $\phi$ for $f_{-1}$ and $S$ for $\bigwedge_{i} \mathfrak{o}\left(a_{i}\right)$, we obtain

$$
\phi(S)^{\# \#} \subseteq \phi\left(S^{\# \#}\right)=\phi(\mathfrak{o}(a))=\mathfrak{o}(h(a)) \subseteq \bigcap_{i} \mathfrak{o}\left(h\left(a_{i}\right)\right)=\bigcap_{i} \phi\left(\mathfrak{o}\left(a_{i}\right)\right)=\phi(S),
$$


in short

$$
\phi(S)^{\# \#} \subseteq \mathfrak{o}(h(a)) \subseteq \phi(S) .
$$

Since for a complemented $C, C^{\# \#}=C$, this makes $\phi(S)^{\# \#}=\mathfrak{o}(h(a))$ and the statement follows.

6.3. Recall from III.7.3 in [15] that a localic map $f: L \rightarrow M$ is closed if the image of each closed sublocale is closed (this concept captures the closedness of continuous maps) and that when this is so then $f[\mathfrak{c}(a)]=\mathfrak{c}(f(a))$ for each $a \in L$. Further, $f$ is closed if and only if, for its left adjoint $h$,

$$
f(a) \vee b=f(a \vee h(b)) \text { for every } a \in L \text { and } b \in M \text {. }
$$

6.3.1. Proposition. A closed localic map preserves all exact meets.

Proof. Let $f: L \rightarrow M$ be a localic map. The image function $f[-]: \mathcal{S} \ell(L) \rightarrow \mathcal{S} \ell(M)$ is a left adjoint and hence it preserves suprema. Thus we obtain

$$
\bigvee \mathfrak{c}\left(f\left(a_{i}\right)\right)=\bigvee f\left[\mathfrak{c}\left(a_{i}\right)\right]=f\left[\bigvee \mathfrak{c}\left(a_{i}\right)\right]=f[\mathfrak{c}(a)]=\mathfrak{c}(f(a))
$$

6.3.2. An interesting consequence of this fact is the extension to frames of the result of E. Michael [14, Corollary 1] that the image of a (normal or regular) paracompact space under a continuous closed mapping is paracompact (see also [9, Corollary to Theorem 2]). For observing that, recall from [9] that a subset $U$ of $L$ is a closed covering if $x=\bigwedge_{u \in U}(x \vee u)$ for every $x \in L$. A closed covering is a dual-refinement [8] of a cover $A$ if for each $u \in U$ there exists $a \in A$ such that $u \vee a=1$. By Theorem 1 of [9] (cf. [8, Theorem 3.3]), a frame $L$ is paracompact and normal iff each cover $A$ of $L$ has a conservative dual-refinement.

Corollary. The image of a normal paracompact frame under a closed localic mapping is paracompact.

Proof. Let $L$ be a normal paracompact frame and $f$ be a closed localic onto mapping from $L$ onto a frame $M$; we denote by $h$ its left adjoint. To prove that $M$ is paracompact, it suffices, by the mentioned result of Dowker-Strauss [9], to show that every cover of $M$ has a conservative dual-refinement. Let $C$ be a cover of $M$. Then $A=h[C]$ is a cover of $L$ and by hypothesis there is a conservative dual-refinement $U$ of $A$. Let $V=f[U]$. By Proposition 6.3.1, $V$ is conservative. Moreover, it is a closed covering of $M$ : for each $y \in M$,

$$
y=f(x)=f\left(\bigwedge_{u \in U}(x \vee u)\right)=\bigwedge_{u \in U} f(x \vee u) \geqslant \bigwedge_{u \in U} f(x) \vee f(u)=\bigwedge_{v \in V}(y \vee v) .
$$


Finally, it is a dual-refinement. Indeed, for each $v=f(u) \in V$, let $a \in A$ be such that $a \vee u=1$ and consider $c \in C$ such that $a=h(c)$. Since $f$ is closed, we may conclude by (6.3.1) that $v \vee c=f(u) \vee c=f(u \vee h(c))=f(u \vee a)=1$.

Acknowledgements. We are indebted to the referees for several helpful comments that have improved the presentation of the paper. We further thank

J. Gutiérrez García for some useful discussions that led to a shortening of our original proof of Theorem 3.4.1.

\section{REFERENCES}

[1] C. E. Aull and W. J. Thron, Separation axioms between $T_{0}$ and $T_{1}$, Indag. Math. 24 (1963) $26-37$.

[2] R. N. Ball, Distributive Cauchy lattices, Algebra Universalis 18 (1984) 134-174.

[3] R. N. Ball, J. Walters-Wayland and E. Zenk, The P-frame reflection of a completely regular frame, Topology Appl. 158 (2011) 1778-1794.

[4] B. Banaschewski and A. Pultr, Variants of openness, Appl. Categ. Structures 2 (1994) 331350.

[5] B. Banaschewski and A. Pultr, Pointfree aspects of the $T_{D}$ axiom of classical topology, Quaest. Math. 33 (2010) 369-385.

[6] G. Bruns, Darstellungen und Erweiterungen geordneter Mengen II, J. für Math. 210 (1962) $1-23$.

[7] G. Bruns and H. Lakser, Injective hulls of semilattices, Canad. Math. Bull. 13 (1970) 115118.

[8] X. Chen, On the paracompactness of frames, Comment. Math. Univ. Carolinae 33 (1992) 485-491.

[9] C. H. Dowker and D. Papert, Paracompact frames and closed maps, Symp. Math. 16 (1975) 93-116.

[10] M. J. Ferreira and J. Picado, On point-finiteness in pointfree topology, Appl. Categ. Structures 15 (2007) 185-198.

[11] K.H. Hofmann and J. D. Lawson, The spectral theory of distributive continuous lattices, Trans. Amer. Math. Soc. 246 (1978) 285-310.

[12] J. R. Isbell, Atomless parts of spaces, Math. Scand. 31 (1972) 5-32.

[13] P. T. Johnstone, Stone Spaces, Cambridge Studies in Advanced Mathematics 3 (Cambridge University Press, Cambridge 1982).

[14] E. Michael, Another note on paracompact spaces, Proc. Amer. Math. Soc. 8 (1957) 822-828.

[15] J. Picado and A. Pultr, Frames and Locales (topology without points), Frontiers in Math. 28 (Springer, Basel 2012).

[16] T. Plewe, Sublocale lattices, J. Pure Appl. Algebra 168 (2002) 309-326.

[17] A. Pultr, Frames, in: M. Hazewinkel (Ed.), Handbook of Algebra, Vol. 3 (Elsevier, 2003), pp. 791-857.

[18] J. Todd Wilson, The Assembly Tower and Some Categorical and Algebraic Aspects of Frame Theory, PhD Thesis, Carnegie Mellon University, 1994. 
Department of Mathematics, University of Denver, Denver, CO 80112, U.S.A.

E-mail address: rball@du.edu

CMUC, Department of Mathematics, University of Coimbra, 3001-501 Coimbra, PORTuGAL

E-mail address: picado@mat.uc.pt

Department of Applied Mathematics and iti, MFF, Charles University, Malostranské nám. 24, 11800 Praha 1, Czech Republic

E-mail address: pultr@kam.ms.mff.cuni.cz 